EPJ Web of Conferences 59, 05020 (2013)

DOI: $10.1051 /$ epjconf/20135905020

(C) Owned by the authors, published by EDP Sciences, 2013

\title{
Multiple scattering of slow ions ion a partially degenerate electron fluid
}

\author{
G. Maynard ${ }^{\mathrm{a}}$ and C. Deutsch \\ Laboratoire de Physique des Gaz et des Plasmas, CNRS-Université Paris-Sud, Bât. 210, \\ 91405 Orsay, France
}

\begin{abstract}
We extend former investigation of the transport of an energetic ions beam through a partially degenerate plasma. In the present work we investigate the electron contribution to the angular dispersion of the beam in the low velocity regime (ion beam velocity less than the average electron velocity). The multiple scattering contribution is implemented within the linear response function through an analytic and mean field interpolation of the target electron dielectric function between $\mathrm{T}=0$ (Lindhard) and $\mathrm{T} \gg \mathrm{T}_{\mathrm{F}}$ (Fried-Conte). New simple analytical formulas are derived for the average scattering angle in the limiting cases of small and high temperature and also an interpolating formula is proposed at any degeneracy.
\end{abstract}

\section{INTRODUCTION}

The interaction of energetic ions beams with a dense target is governed by the energy loss cross section and by multi scattering (MS) collisions. At high energies, $E_{B} \gg 1 \mathrm{MeV}, E_{B}$ being the energy of the beam ions, the main effect of MS collisions is to increase the beam emittance. This should be taken into account, for example, when analysing fast ignition with proton beams [1]. MS collisions can also have a significant contribution to isochoric heating of dense plasmas by reducing the linear range of the beam particles. In high- $Z$ targets, ion-ion collisions yield the main contribution to elastic scattering. However for low-Z targets, and in particular in hydrogen or D-T plasmas, electron-ion collisions can have a significant contribution. In the present work we are focussing on the calculation of MS in the low velocity regime, close to the maximum of the stopping cross section, where the coupling between the beam particles and the plasma electrons is maximum. In connection with fast ignition, we consider fully ionized light projectiles, $Z_{B} \leq 6, Z_{B}$ being the charge of the beam ions. At these low charges, and for high density plasmas, the interaction of the projectile with the target can be described within the linear response theory. MS cross sections in a fully degenerate electron jellium at $T=0$ have been studied in [2]. This work has been recently extended to any temperature using the general Random Phase Approximation (RPA) formalism [3]. Using this formalism, new analytic formulas of MS on electrons have been derived. In the following section we give a brief presentation of the MS cross section within the RPA. The analytic formulas are then given and compared to numerical results. Atomic units are used.

\section{THEORY}

Let consider an energetic ion beam of charge $Z_{B}$, mass $M_{B}$, velocity $V_{B}$ and energy $E_{B}$ interacting with a plasma of temperature $T$ and electron density $n_{e}$ leading to a Fermi temperature $T_{F}$. The energy of the beam ions is much larger than the average energy of the plasma electron : $E_{B} \gg T_{F}, T$. Here, we

\footnotetext{
ae-mail: gilles.maynard@u-psud.fr
}

This is an Open Access article distributed under the terms of the Creative Commons Attribution License 2.0, which permits unrestricted use, distribution, and reproduction in any medium, provided the original work is properly cited. 


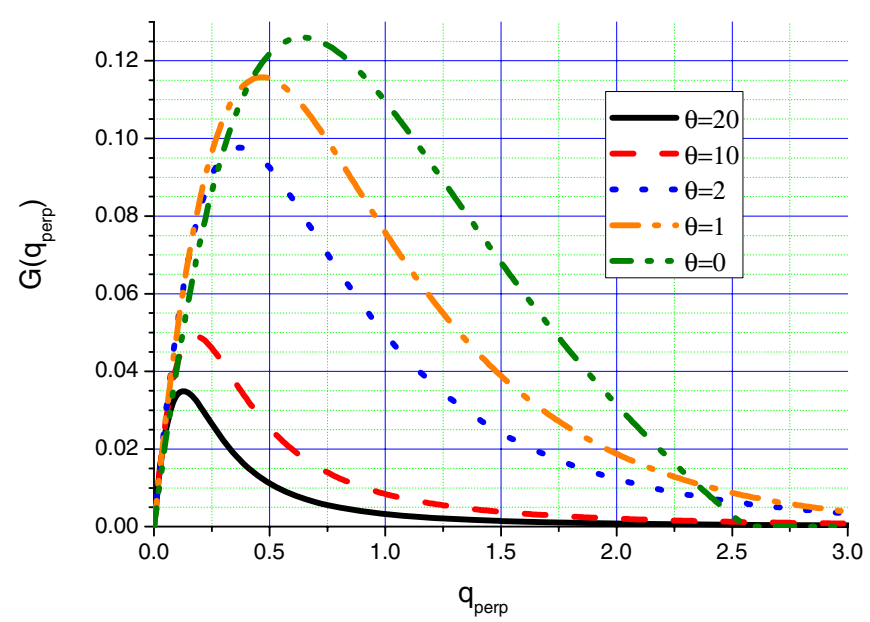

Figure 1. $G\left(q_{\text {perp }}\right)$ versus $q_{\text {perp }}$, in atomic units, at various temperatures.

consider the low velocity regime $V_{B}<V_{t h} \approx \sqrt{2\left(T+T_{F}\right)}$. Quite generally, the stopping of the beam is due to ion-electron (free or bound) collision while the transverse dispersion mainly comes from ion-ion collisions. However, for hydrogen or helium plasma ion-electron collisions can contribute significantly to the transverse dispersion. In such cases it is important to have a robust estimation of the influence of angular scattering. In particular, simple analytic formulas of angular scattering processes are required to perform numerical simulations of beam-plasma interaction.

In the linear approximation, the ion-electron potential is derived from the dynamic response function $[2,3]$. In order to consider any degeneracy values, we have used the full Random Phase Approximation (RPA) of this quantity. In the low velocity regime it reads

$$
S(q, \omega)=\frac{2 q \omega}{\left(q^{2}+q_{S}^{2}\right)^{2}} \cdot \frac{1}{1+\exp \left(\frac{q^{2}}{8 T}-\eta\right)},
$$

where $\eta$ is the electron degeneracy, and $q_{s}$ is given by :

$$
q_{s}=\frac{1}{2} q_{T F}^{2} \theta^{1 / 2} F_{-1 / 2}(\eta),
$$

with $q_{T F}$ the Thomas-Fermi screening parameter, $\theta=T / T_{F}$ and $F_{v}(\eta)$ is the Fermi function.

\subsection{Single Scattering}

From the expression of the response function one can derive the differential scattering cross section for perpendicular momentum transfer:

$$
\begin{aligned}
& \frac{d P}{d q_{\text {perp }}}=V_{B} G\left(q_{\text {perp }}\right) \\
& G\left(q_{\text {perp }}\right)=4 Z_{B}^{2} q_{\text {perp }} \int_{q_{\text {perp }}}^{\infty} \frac{d q}{\left(q^{2}+q_{S}^{2}\right)^{2}} \cdot \frac{1}{1+\exp \left(\frac{q^{2}}{8 T}-\eta\right)} .
\end{aligned}
$$

In Figure 1 we have reported $G\left(q_{\text {perp }}\right)$ versus $q_{\text {perp }}$ for a high density plasma, $n_{e}=5 \times 10^{23} \mathrm{~cm}^{-3}$. One can observe that for low momentum transfer, $G$ is little affected by the temperature, while it decreases with $T$ at intermediate values of $q_{\text {perp }}$ and increases with $T$ for the highest value of $q_{p e r p}$; this limiting case is more clearly seen in Figure 2. 


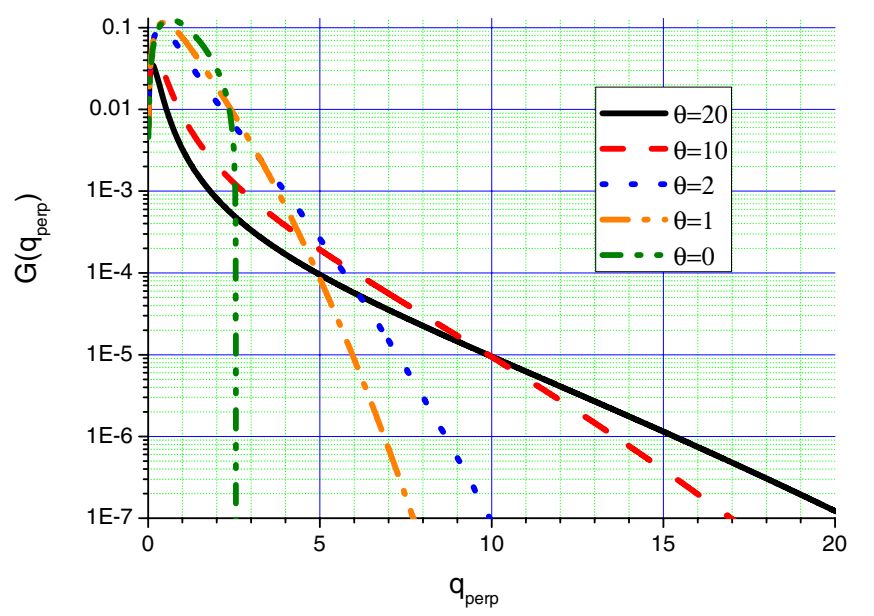

Figure 2. Same as Figure 1.

\subsection{Multiple scattering}

The angular distribution function of the beam ions after flowing through a distance $X, f(\alpha, X)$, can be expressed as $f(\alpha, X)=\int_{0}^{\infty} \kappa J_{0}(\kappa \alpha) \exp \left[-X n_{e} \sigma(\kappa)\right] d \kappa$, the cross section $\sigma$ being given by $n_{e} \sigma(\kappa)=$ $\int_{0}^{\infty}\left(1-J_{0}\left(\kappa q_{\text {perp }} / M_{B} V_{B}\right)\right) G\left(q_{\text {perp }}\right) d q_{\text {perp }}$ [4]. Due to the large difference between ions and electrons masses and energies, the scattering angle encountered by the beam ions in one collision is quite small, $\left(\kappa q_{\text {perp }} / M_{B} V_{B}\right) \ll 1$, therefore one gets $n_{e} \sigma(\kappa) \approx \kappa^{2} \bar{\theta}^{2} / 4$, with $\bar{\theta}^{2}=\bar{q}_{\text {perp }}^{2} /\left(M_{B} V_{B}\right)^{2}$ where $\bar{q}_{\text {perp }}$ is the average momentum transfer in one collision. $f(\alpha, X)$ then becomes the usual Gaussian distribution, $f(\alpha, X)=2 \exp \left[-\alpha^{2} / \bar{\alpha}^{2}\right] / \bar{\alpha}^{2}$ with

$$
\begin{aligned}
& \bar{\alpha}^{2}(X)=\int_{0}^{\infty} \alpha^{2} f(\alpha, X) \alpha d \alpha \\
= & X \bar{\theta}^{2}=X \bar{q}_{\text {perp }}^{2} /\left(M_{B} V_{B}\right)^{2} .
\end{aligned}
$$

The multiple scattering processes can then be determined from the knowledge of $\bar{\alpha}$ for which analytical expressions are given in the next section.

\section{ANALYTICAL FORMULAS}

The average scattering angle obeys the scaling relation $\bar{\alpha}^{2}=\left[X n_{e} Z_{B}^{2} /\left(M_{B}^{2} V_{B}^{2}\right)\right] U\left(T, n_{e}\right)$ with $U\left(T, n_{e}\right)=\left(\sqrt{8 T} / \pi n_{e}\right) \int_{0}^{\infty} \frac{u^{4}}{\left(u^{2}+u_{s}^{2}\right)^{2}} \frac{d u}{1+\exp \left[u^{2}-\eta\right]}, \quad u_{s}^{2}=q_{s}^{2} / 8 T$. The function $U$ has simple analytic expressions at low and high temperatures, from which we have determined an interpolation formula:

Low temperature, $T / T_{F} \ll 1$ :

$$
U\left(T, n_{e}\right)=\frac{\sqrt{8 T} u_{s}}{\pi n_{e}}\left[\delta-\frac{3}{2} \tan ^{-1} \delta+\frac{\delta}{2\left(\delta^{2}+1\right)}\right], \quad \delta=\frac{\sqrt{\eta}}{u_{s}}
$$




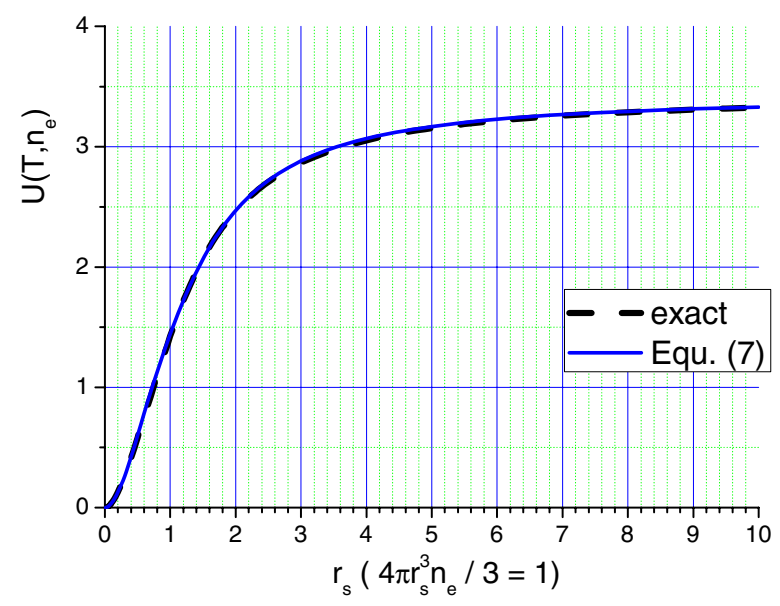

Figure 3. The function $U$ in terms of the scaled electron density at a temperature of $50 \mathrm{eV}$.

High temperature, $T / T_{F} \gg 1$ :

$$
U\left(T, n_{e}\right)=\frac{2 \pi}{T}\left[1-\alpha_{1} \sqrt{\frac{u_{s}^{2}}{u_{s}^{2}+\beta_{1}}}-\alpha_{2} \sqrt{\frac{u_{s}^{2}}{u_{s}^{2}+\beta_{2}}}\right],
$$

with $\alpha_{1}=0.229, \alpha_{2}=1-\alpha_{1}, \beta_{1}=0.04, \beta_{2}=0.382$.

Interpolating formula, valid at any temperature:

$$
U\left(T, n_{e}\right)=U_{0}\left[1-\alpha_{1} \sqrt{\frac{u_{s}^{2}}{u_{s}^{2}+\beta_{1}}}-\alpha_{2} \sqrt{\frac{u_{s}^{2}}{u_{s}^{2}+\beta_{2}}}\right],
$$

with $U_{0}=\frac{\sqrt{2 T}}{\pi n_{e}} F_{-1 / 2}(\eta)$. A comparison between the exact values and Eq. (7) results is reported in Figure 3. We can observe in this figure that the interpolating formula is in close agreement with the numerical result on the whole range of the electron degeneracy.

\section{CONCLUSION}

An accurate analytic formula, Eq. (7), has been derived for fast calculations of the electron contribution to the multiple scattering effect in the transport of an energetic ion beam through a high density plasma. This formula smoothly interpolates between the classical high temperature limit, Eq. (6), up to the strongly degenerate regime, Eq. (5), so that it can be applied at any plasma degeneracy provided that the ion velocity remains less than the average velocity of the plasma electrons.

This work was supported in part by Euratom (contract Euratom-CEA V3599.001).

\section{References}

[1] G. Maynard and M.D. Barriga-Carrasco, Nucl. Instrum. Meth. Phys. Res. A 544, 84 (2005)

[2] C. D. Archubi and N.R. Arista, Phys. Rev. A 72, 062712 (2005)

[3] R. Popoff, G. Maynard and C. Deutsch, Phys. Rev. E 80, 046408 (2009)

[4] H. A. Bethe, Phys. Rev. 89, 1256 (1953) 